\title{
Agent based data collecting in forest fire monitoring system
}

\author{
Ljiljana Bodrozic, Darko Stipanicev, \\ Department for Modeling and Intelligent Systems \\ FESB - Faculty of Electrical Engineering, Machine Engineering and Naval Architecture \\ UNIVERSITY OF SPLIT, 21000 Split, CROATIA, R.Boskovisa bb \\ Web: http://laris.fesb.hr Contact e-mail: lijiljana@fesb.hr
}

\begin{abstract}
: $\mathbf{2 4}$ hour surveillance is of most importance in forest fire protection systems. Real time data must be acquired to have reaction fast enough to suppress fire occurrence or minimize damage made by forest fires.
\end{abstract}

In forest fire monitoring systems, usually a large area must be controlled. Real time data have to be collected and processed in time. When amount of data reaches critical volume, modern software techniques have to be implemented in order to accomplish system goals.

In this work we have implemented the agent technology on data retrieval and processing. Multi agent system for real time data collection and processing is described.

This work is a part of more complex integral project of forest fire protection in Split and Dalmatia County. The integral forest fire protection system will be based on information system for integration of all activities connected with early fire detection by 24 hours video and meteorological monitoring, management of forest fire fighting and post-fire recuperation of burned landscape.

\section{INTRODUCTION}

Forest fire management is of large importance in region of Split in Dalmatia County where tourism and agriculture are traditionally primary economy branches. Forest fire management could be done in three phases:

- before fire, focusing on fire prevention,

- $\quad$ during fire as a decision support system in fire fighting strategy and

- $\quad$ after fire for planning the best recovery strategy.

The system input parameters for could be grouped into four categories:

- fuel characteristics

- $\quad$ terrain topology

- meteorological data and

- $\quad$ images (video data).

First two parameters are characteristic of area of interest and are not changed frequently, so we store them as a GIS (Geographic Information System) layers. Meteorological data and images are parameters that changes over space and time with relatively high frequency. The change of meteorological data could be recognized in hour scale, and the change of image data, taking into account only information connected with forest fires, in minute scale. Also for forest fires prediction system, the history of meteorological data (archive values) are quite important. In order to monitor meteorological parameters and collect images in real time, the sensory network has to be established.

In our approach, which is now in developed for Split and Dalmatia County and called Integral Forest Fires Monitoring System [1], the system is TCP/IP based system conceived of:

- $\quad$ sensory networks and

- central server units for collecting, processing and storing all data.

Each sensory network has few sensory (monitoring) units, and each unit includes:

- $\quad$ pan / tilt / zoom controlled video camera connected to network embedded video Web server,

- mini meteorological stations connected to network embedded data web servers, and

- $\quad$ wireless (IEEE $802.11 \mathrm{~b} / \mathrm{g}$ ) communication unit.

The system overview is shown in figure 1 .

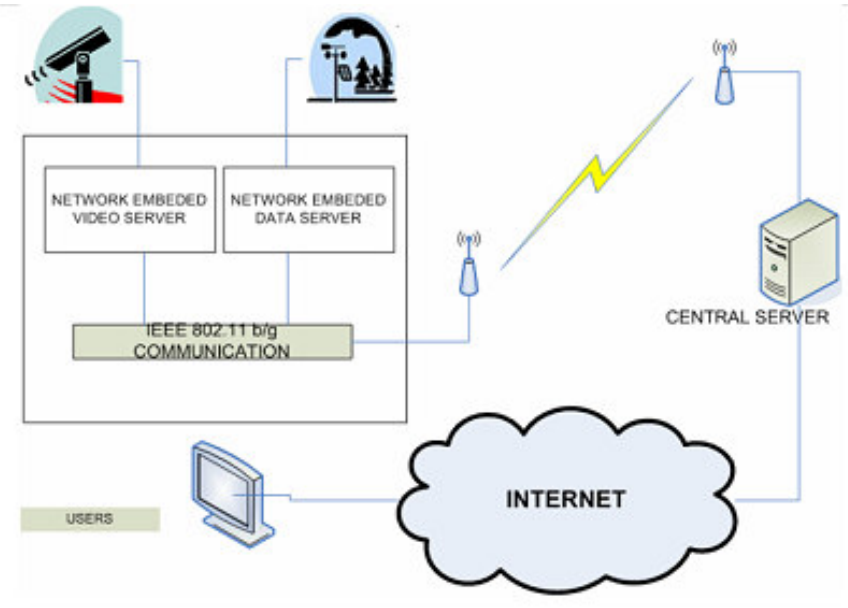

Figure 1 - The concept of Split and Dalmatia County Integral Forest Fire Monitoring System 
Figure 2 shows the photo of one experimental monitoring unit which was in function during summer season 2005.

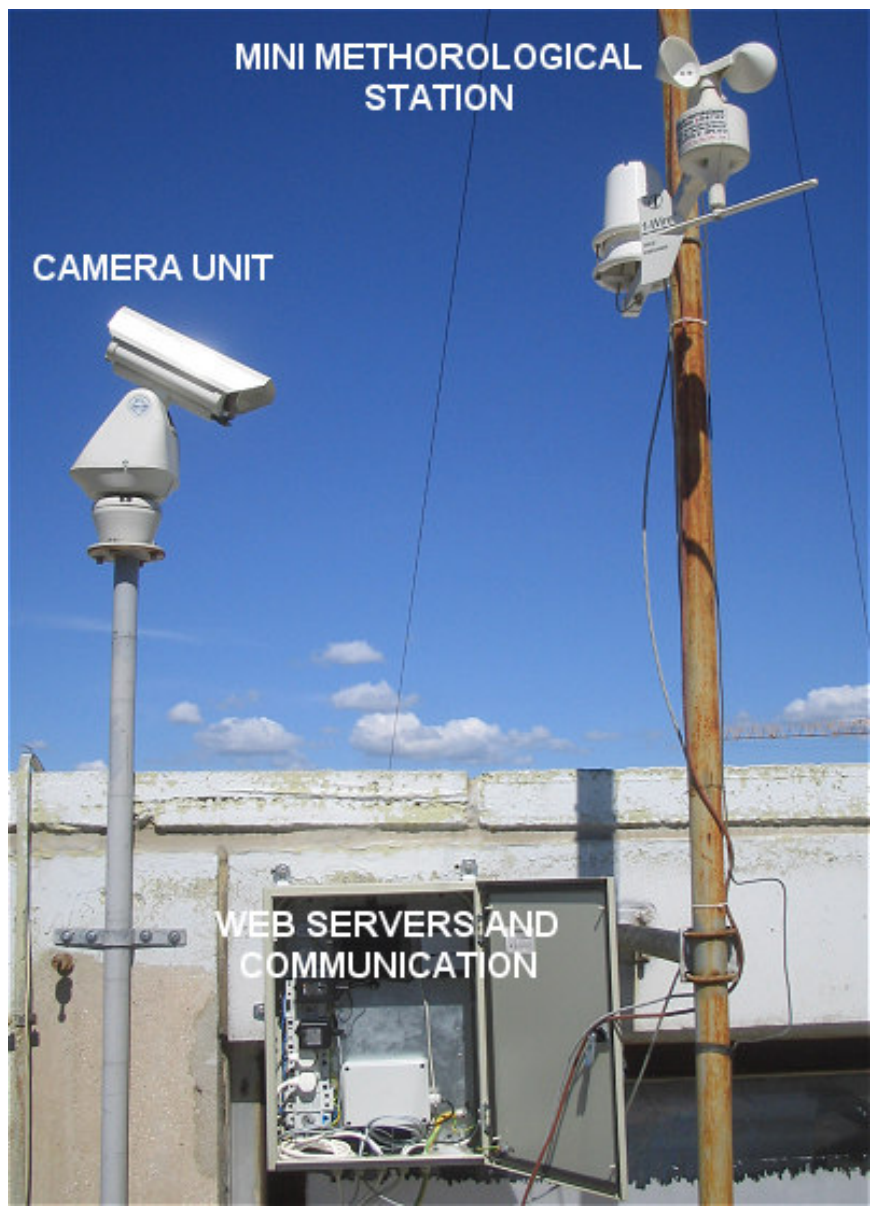

Figure 2 - Experimental sensory (monitoring) unit

Meteorological data and images collection could be done by two methodologies:

- By central server pull or

- $\quad$ By embedded servers push

In Integral Forest Fire Monitoring System both methodologies have been applied, the first one for cyclical image collecting every 5 seconds and meteorological data collecting every 10 minutes and former for alarming if alarm values of certain meteorological or system parameters are reached.

The novelty of our approach described in this paper is the application of agent based technologies for both tasks. The multi-agent based system was developed and implemented for meteorological and video data collecting (pulling) by central server, but also for pushing data by sensory unit if measured real time data reach the alarm values.

\section{MULTI AGENT SYSTEM FOR METEOROLOGICAL AND VIDEO DATA COLLECTING}

Application of agent oriented methodologies in process monitoring and control is relatively new approach particularly suitable for distributed and dislocated systems $[2,3,4]$.

Formally agent could be defined as [5] :

“... an encapsulated computer system, situated in some environment, and capable of flexible autonomous action in that environment in order to meet its design objectives"

Agents designed for forest fire monitoring system followed strictly these guidelines, because the system was conceived as a modular system where each module is autonomous, aware of its environment and capable for active behavior if alarmed.

Environment awareness is accomplished by connecting numerous meteorological sensors to a network embedded microcontroller unit. Network embedded microcontroller unit was responsible for collecting data from sensors, formatting and preprocessing them and giving them to the central server agent when asked for.

The network embedded microcontroller unit is Ethernet enabled and runs a simple web server application so agent communication was realized by http connections. First data preprocessing (digitalization) was done by the sensor itself. After that, microcontroller unit made further data processing, data averaging in 10 minutes intervals and data formatting into a format understandable by central server agent. At the begging that was XML format, but to minimize the activity of embedded microcontroller unit at the end the direct agent communication and exchange variable values was adopted. Agent deployed in a central server communicates with an embedded device over a network, asking for formatted data by http request. The important is to emphasize that agents communicate using standard FIPA ACL (Agent Communication Language).

The same procedure was used for image data collecting. The video part of monitoring unit had pan / tilt /zoom controlled heavy duty video camera and embedded video Web server. The embedded video Web server was responsible for image digitalization and compression in JPEG format. Each camera had a number of preset positions to cover all monitoring unit surrounding. The Camera Agent on central server was responsible not only for image collecting but also for moving camera to appropriate preset position by adjusting camera's pan and tilt angles. Also in this case all communication between Camera Agent and video Web server was done by http requests, but image transfer was realized by ftp requests.

The fundamental agent based is shown in Figure 3 


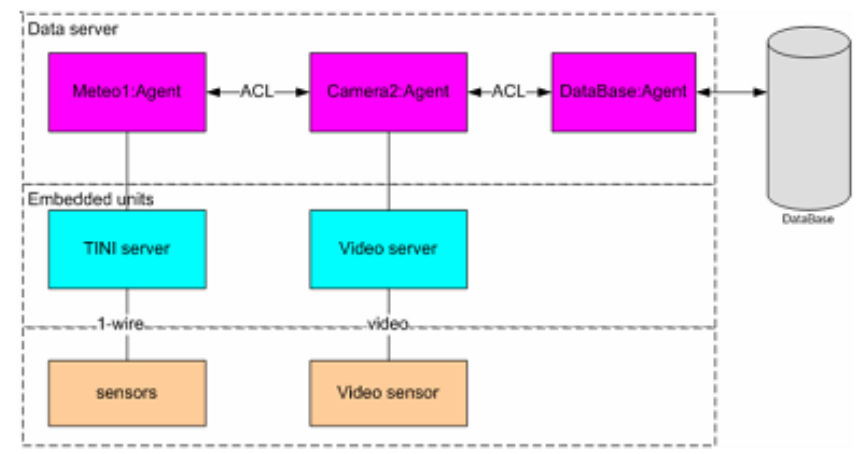

Figure 3 - The fundamental system architecture

In figure 3 there is a third agent called DataBase Agent too. His task was communication with data base - storing meteorological data and writing notes about collected images and alarms.

Two types of alarms could be encountered - the simple one meteorological data alarms and the complex one forest fires alarms. Meteorological alarms are generated by simple expert system, and forest fires alarms are generated by complex forest fires recognition algorithm. The algorithm was originally developed for our system and based on recognition of smoke caused by possible forest fire analyzing the images collected by Camera Agents. This algorithm will be subject of another paper. In this one we concentrate only on agent based data collecting system.

The user (operator) communicates with the system by the assistance of User Agent and the User Agent retrieve data from the data base by the assistance of DataBase Agent. Typical user screen is shown in figure 4.

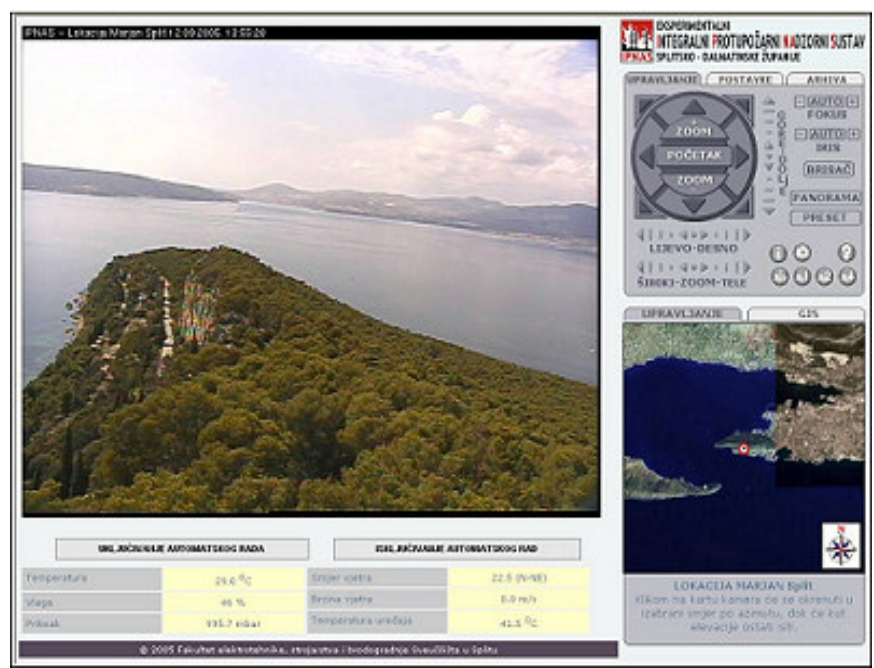

Figure 4. Typical user interface (location Marjan Hill Split)
The main advantage of the agent based approach for data collecting is its simple extension for distributed systems. The forest fire monitoring system of Split and Dalamtia County will be a sensory network system. According to our calculations for covering all the county about 50 monitoring units have to be installed, and to cover the most vulnerable areas about 25 units will be needed. Because of that the system is designed as a distributed system, without one central unit. Each area, for example island Brac, will have its own central server, but all of them will be mutually connected through TCP/IP network. Figure 5 shows the distributed system overview.

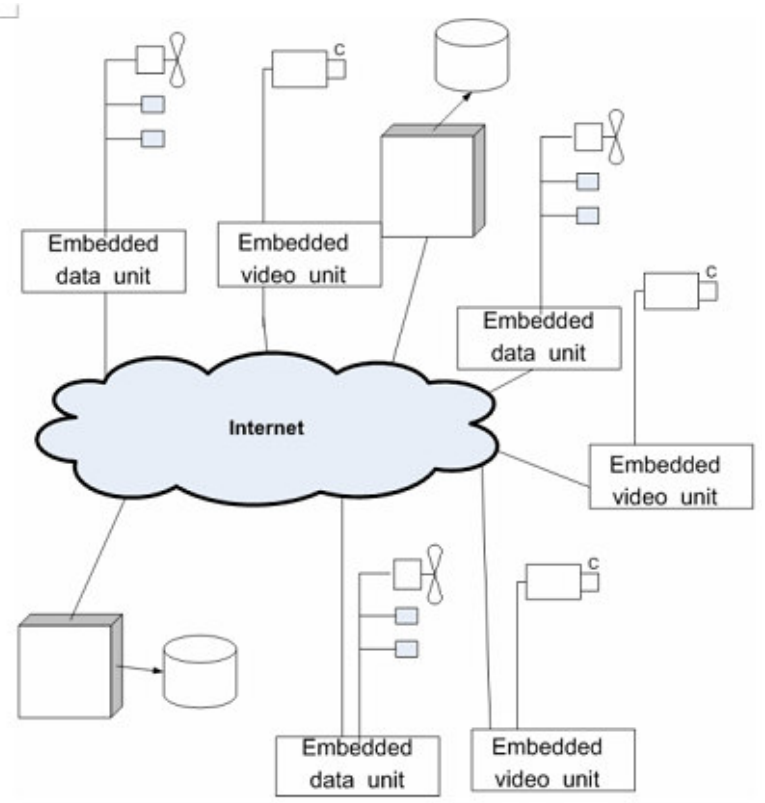

\section{Figure 5 - Distributed system with few monitoring stations and central units}

Adding new monitoring unit or server unit could be easily realized by simple adding new agents. The distributed agent name server will be used to simplify agent communication. Experimental system which was in operation during summer 2005. had three monitoring units and one central processing unit. More details of this system are given in the next chapter.

\section{EXPERIMENTAL SYSTEM DESCRIPTION}

Although our tendency was to create system that would not depend on hardware and software platform, we had to choose test platform for our experimental system.

In variety of sensors available on market, low cost iButton 1-wire sensors were chosen for meteorological data sensing. Together with Dallas TINI embedded microcontroller [6] , this network embedded meteorological station forms a reliable measurement unit. 
Reasons for choosing TINI were its simplicity, low power consumption, open source software support and particularly java interface, because our multi agent based system was developed using JADE (Java Agent Development Framework) [7] as a development framework.

To unify the approach the video camera connected to a video Web server was considered to be a video sensor, the same way as temperature measuring element connected to data Web server was considered to be a temperature sensor. To cover the whole surrounding space each camera had 8 preset positions, and each preset position was considered to be its own video sensor responsible for monitoring certain part of landscape. This means that each camera was treated as 8 independent fixed cameras mounted on the same place. Because of its behavior complexity, data from this camera are taken in two steps. A supervisor agent for pan/tilt/zoom camera is taking care of positioning the camera in sequent ional queue and after the camera is still in place, taking the image from video input.

The supervisor agent is called CameraAgent and agents he controls are called PresetAgent. The sequence diagram of a working system is given in figure 6

\section{CONCLUSION}

Best fire fighting strategy is prevention of fire. Fire fighting starts before fire even occurs. Integral forest fire monitoring system was designed to help in prevention of forest fires occurrence in Split and Dalmatia County. The system was conceived consisting of sensory units forming sensory network and number of server units for data analyzing, storing and presentation. Each sensory unit had two parts, video sensory device and meteorological parameters sensory device.

In this paper we have described our system responsible for data collecting, both image date used in automatic forest fire detection and meteorological data used in prediction of possible fires and fire fighting management. The novelty of our approach is application of unified agent based methodologies for all data collecting. The multi agent system was designed responsible for communication between server units and monitoring units. The advantage of such approach is better control of overall system because of its modularity, easier system enlargement by adding new sensors units and new type of sensor units, and particularly the ability of collected data validation on different levels. The system conceived of three monitoring units and one server unit was in experimental work during summer 2005.

\section{REFERENCES}

[1] D.Stipanicev, B.Hrastnik, Integral model for Forest Fires Protection in Split-Dalmatia County, FESB Report for Split-Dalmatia County, June 2003 (in Croatian)

[2] S.Bussmann, N.R.Jennings, M.J.Wooldridge, On the identification of agents in the design of production control systems, Agent-Oriented Software Engineering, Springer-verlag, pp.141-162, 2001.

[3] D.Stipaničev, M.Štula, Lj.Bodrožić, Multiagent Based Greenhouse Telecontrol System as a Tool for Distance Experimentation, IEEE Int.Symposium on Industrial Electronics, ISIE - 2005, Dubrovnik, 20-23.06.2005.

[4] M.Štula, D.Stipaničev, Agent Based Methodologies in Distributed Control, Proc.of Int. Conf. CEEPUS Summer School Intelligent Control Systems (ISBN 80-214-2976-3), Brno, Czech Rep. 29.08.-11.09.2005. pp. $222-228$

[5] M.Woldridge, N.Jennings, Intelligent Agents:Theory and Practice, Knowledge Engineering Review, Vol.10, No.2, Cambridge University Press, 1995. ....

[6] TINI - Tiny Internet Inteface - http://www.maximic.com/TINIplatform.cfm

[7] JADE - Java Agent Development Framework http://jade.tilab.com/

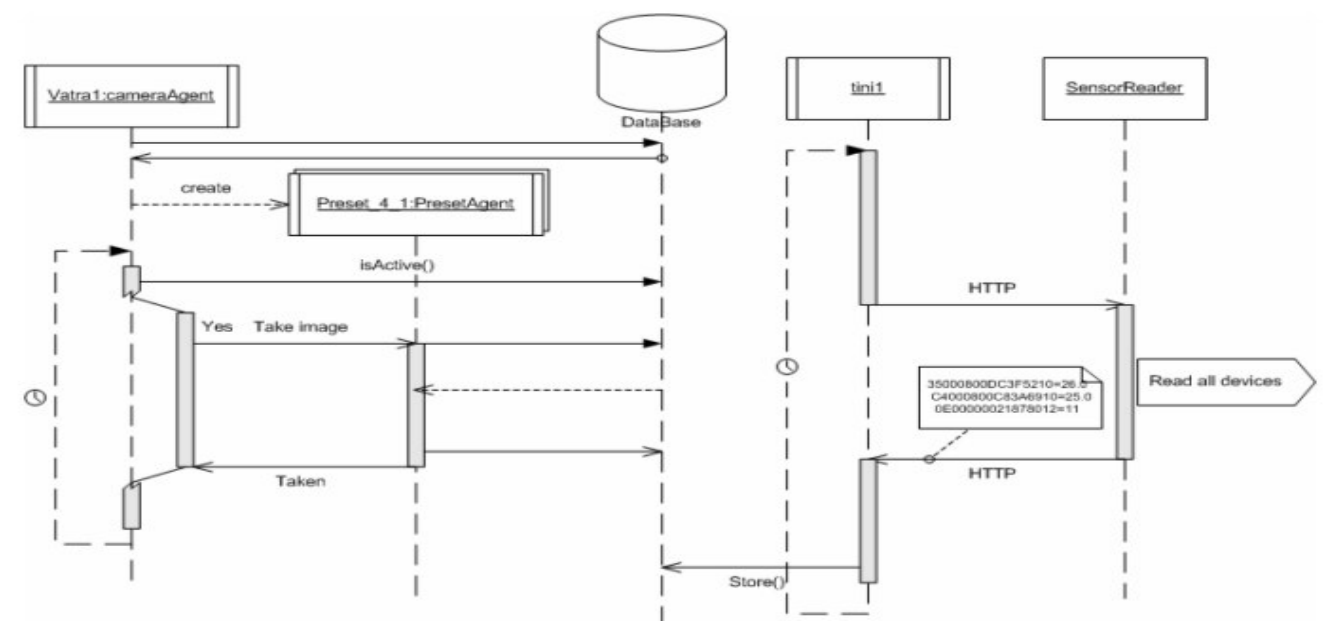

Figure 6 - The agent sequence diagram 